\title{
Developments in Molecular Testing and Biosimilars
}

\author{
Katy Winckworth-Prejsnar, MPH${ }^{\mathrm{a}}$; Elizabeth A. Nardi, MSª Lisa Korin Lentz, $\mathrm{MPH}^{\mathrm{a}}$; \\ Jeffrey A. Crawford, MD ; C. Lyn Fitzgerald, MJa; and Robert W. Carlson, MD ${ }^{\mathrm{a}, \mathrm{c}}$
}

\begin{abstract}
Molecular testing and biosimilars offer the potential for increased access to targeted treatment options and reduction in healthcare costs, but come with significant challenges in ensuring patient access to innovation in cancer care while maintaining safe, effective, ethical, and affordable treatment options. As providers, payers, patients, and the larger healthcare systems become inundated with a wide variety of molecular diagnostics and an increased number of biosimilars coming to market, it will be important to understand regulatory guidance and policy implications relating to the appropriateness of molecular testing and the clinical use of biosimilars in cancer care. In September 2016, NCCN hosted the Molecular Testing and Biosimilars Policy Summit to address the challenges, issues, and opportunities in both the molecular testing and biosimilar landscapes. Keynote presentations and panelists further discussed the status and future of molecular testing and biosimilars within the oncology space, as well as patient access and education needs moving forward.

J Nat/ Compr Canc Netw 2017;15(6):772-782
\end{abstract} doi:10.6004/jncen.2017.0109

\section{Executive Summary}

Molecular testing and biosimilars offer the potential for increased access to targeted treatment options and reduction in healthcare costs, but come with significant challenges to therapeutic decision-making, safety, efficacy, cost, and reimbursement. Increasing healthcare spending and cost pressures are manifesting themselves in complex scientific and clinical debates regarding biosimilars and diagnostics in cancer care. ${ }^{1}$ Although personalized medicine is benefiting patients across a variety of therapeutic areas, the largest proportion of personalized treatment options are within the oncology space. For biosimilars, a biologic that is "highly similar" to an existing FDA-approved biologic drug, there are an estimated 19 pipeline biosimilar molecules in development in the United States. As providers, payers, patients, and the larger healthcare systems become inundated with a wide variety of molecular diagnostics, and an increased number of biosimilars coming to market, it will be

From a National Comprehensive Cancer Network, Fort Washington, Pennsylvania; 'Duke Cancer Center, Duke University, Durham, North Carolina; and 'Fox Chase Cancer Center, Philadelphia, Pennsylvania. Submitted March 17, 2017; accepted for publication April 18, 2017. Dr. Jeffrey Crawford has disclosed that he is a scientific advisor for Merck, Novartis, and Pfizer; is a data safety monitoring board member at Celgene, G1 Therapeutics, Janssen, Merrimack, and Roche; and is a principal important to establish and understand regulatory guidance and policy implications relating to the appropriateness of molecular testing and the clinical use of biosimilars in cancer care. The widespread adoption and payment of molecular testing and biosimilars will require stakeholders to shift away from reliance on traditional clinical trials toward an understanding of molecular data in different and novel ways.

To explore how molecular testing and biosimilars have evolved, NCCN convened the NCCN Policy Summit: Developments in Biosimilars and Molecular Testing in Washington, DC, on September 16, 2016. Stakeholders gathered to discuss the status and future of molecular testing and biosimilars within the oncology space, as well as patient access and education needs. The program consisted of presentations and 2 roundtable discussions with vigorous dialogue and time for audience comments and questions (see supplemental eAp- 
pendix 1, available with this article at INCCN.org, for a list of speakers and panelists).

\section{Developments in Molecular Testing: Benefits, Risks, and Challenges of Application in Cancer Care}

Precision medicine continues as an emerging approach for disease prevention and treatment that considers individual variability in genes, lifestyle, and environment. The advent of precision medicine began with the Philadelphia chromosome found in most patients with chronic myeloid leukemia (CML) and the use of imatinib to treat it. The discovery of imatinib and the precise targeting of the Philadelphia chromosome transformed the care of patients with CML, provided survival benefit, and improved quality of life. ${ }^{2}$ Researchers continue to search for genetic markers that predict likelihood of response to therapy, and the oncology community relies on tests detecting the biomarkers and corresponding targeted therapies to ensure patients receive appropriate cancer treatment specific to the individual characteristics of their particular disease.

Advances in precision medicine continue to transform the way patients with cancer are diagnosed and treated. Patients with cancers such as lung, colon, and breast cancers and melanoma often undergo molecular testing to understand genetic and other molecular information. Once the biomarker makeup is understood, providers may select therapies that target specific biomarkers or mutations. There is a growing library of biomarkers that have been identified, with a growing pharmacopeia available to target them.

The promise of precision medicine has moved to the forefront of national healthcare discussions, leading to several government-led initiatives. President Obama launched the Precision Medicine Initiative in his 2015 State of the Union address, followed by the White House Cancer Moonshot in his 2016 State of the Union address. In mid-December 2016, President Obama signed the 21st Century Cures Act, which contains $\$ 4.8$ billion to advance these initiatives. Part of these government-led initiates is to establish mechanisms to aggregate data and break down data silos in order to establish broad utility for precision medicine. These efforts strive to drive research, technology, and medical advances in precision medicine.
Additionally, the Health and Medicine Division of the National Academies of Sciences, Engineering, and Medicine appointed a committee of experts to examine how providers, regulators, payers, and test developers can assure timely access to tests linked to targeted treatments, while also ensuring quality and safety of tests, including avoidance of poorly validated or inappropriate tests. In March 2016, in its report Biomarker Tests for Molecularly Targeted Therapies: The Key to Unlocking Precision Medicine, the committee issued recommendations about regulatory reimbursement policy, clinical practice issues, and data challenges to guide precision medicine in advancing the care of patients with cancer and other diseases. ${ }^{3}$

\section{Enablers and Barriers in Precision Medicine}

Knowledge of cancer biology is becoming less of a barrier to precision medicine as genetic variants and biological networks are identified and documented. The challenge is understanding the information, what the true drivers of disease are, and how they relate. Technology can act as an enabler of precision medicine and has advanced to where there is an opportunity to sequence the whole genome for cancer, with costs decreasing and insurers providing coverage as a benefit for patients. ${ }^{2}$ Technology can solve some of the common barriers to testing, such as insufficient tissue sample, through multiplex testing and blood-based assays. ${ }^{4}$ Additionally, targeted therapies specific to cancer drivers are becoming a leading area of healthcare spending. Between 2003 and 2013, the percentage of targeted medicines making up the total sales within the oncology market increased from $11 \%$ to $46 \%$, and $73 \%$ of therapies in the cancer pipeline have the potential to be used as precision medicine. ${ }^{5,6}$ These enablers of precision medicine-technology, knowledge of cancer biology, and increasing pharmacopeia of effective targeted therapies-will likely eventually lead to a patient experience involving sequencing at diagnosis, understanding the genetic makeup of the tumor, and making the best care judgements and decisions based on that knowledge. ${ }^{2}$

With the advancement in knowledge and technology, patients, providers, payers, and the healthcare system are inundated with a wide variety of purveyors of genomic tests. Ensuring the quality of a test is principal; assessing such quality is a challenge 
Winckworth-Prejsnar et al

for physicians, who are key in ordering these tests and making decisions based on them. Additionally, highly publicized examples of unfounded claims regarding analytic validity, such as Theranos, Inc. (Palo Alto, CA) and Pathway Genomics (San Diego, $\mathrm{CA}$ ), and subsequent regulatory actions created concern that caution and greater regulation are warranted. Independent guidelines, such as those from NCCN, can play a critical role in helping to ensure quality. Ultimately, transparency and accountability will help ensure quality for providers and patients. ${ }^{2}$

Much of the utility of molecular testing is dependent on physicians understanding the test, its results, and using it appropriately in the diagnosis and therapy of a disease. The technologies, the biologic insights, and the therapies are growing at an inordinate rate and oncologists are facing challenges. ${ }^{2}$ Providers are challenged by the need to learn about, understand, and interpret molecular testing, and to stay abreast of developments and therapies and apply these skills when caring for patients. A 2012 study found that $98 \%$ of physicians surveyed supported the view that genetic variations may predict drug response, but only $10 \%$ felt adequately informed about the testing. ${ }^{7}$ Even when tests prove their analytical validity and clinical utility, providers face logistical barriers, such as test reports not being fully integrated into the medical record. Further, national attention and the promise of precision medicine has led to increasing awareness among patients. ${ }^{4}$ Providers are tasked with explaining the complexity of molecular testing to patients, including the possibility that the results may not affect clinical care. If a patient's molecular diagnostic report is linked to therapy, providers need the tools to show and explain the link to patients.

Precision medicine pushes the cancer community to an area often lacking high-level evidence of clinical benefit. ${ }^{4}$ Often, coverage and reimbursement for molecular testing depend on proving clinical utility through a body of evidence. Building a library of evidence for diagnostics, however, has proven challenging on various fronts. For instance, there may be melanoma studies showing that targeting the BRAF gene with certain therapeutic agents provides benefit, but for stomach cancer with the same mutation, there is little evidence or data available. ${ }^{4}$ When constructing a research trial, the number of patients with a specific mutation of a disease type may be small. After screening and consenting, the trial may result in an even smaller sample size. Pharmaceutical companies often take on the cost of developing a diagnostic if a drug cannot obtain regulatory approval without the companion diagnostic; this may involve performing biomarker testing to screen patients for trial participation and often results in negative tests deeming patients ineligible. ${ }^{4}$ There is a disincentive for industry to invest in such trials. Thus, traditional clinical trials often cannot fully build the evidence required for precision medicine.

A basket trial aims to treat patients with therapies that target specific mutations found across disease types, rather than treating by cancer type or site of origin. These trials can make important strides in the overall understanding of biomarkers in cancer care and contribute to precision medicine. BRAF V600 mutations occur in various nonmelanoma cancers, and Hyman et $\mathrm{al}^{8}$ sought to explore the use of vemurafenib outside its indication of melanoma through a basket trial study. The study results found that not all patients across all diseases with this mutation benefit from the drug, but there are groups that do, such as those with non-small cell lung cancer. In order to understand the utility of targeting, one must understand the full disease context. The large body of data and evidence needed to propel precision medicine forward has led to the development of data warehouses or registries that aggregate the experience of multiple institutions. The NCI Genomic Data Commons provides the cancer research community with a unified data repository that enables data sharing across cancer genomic studies in support of precision medicine. Companies such as Syapse, Inc. (Palo Alto, CA) or Foundation Medicine, Inc. (Cambridge, MA) aim to contribute to precision medicine with various services that result in a repository of data. The Molecular Evidence Development Consortium (MED-C) created a registry that will gather genomic-level data on patients with cancer and information about their treatments and clinical outcomes. Even with these efforts, as well as the White House Cancer Moonshot initiative and the 21st Century Cures Act, there is a need for the harmonization of standardized data elements across data platforms to virtually link and integrate data. ${ }^{4}$ Mechanisms to aggregate data and break down data silos are required to establish broad utility for precision medicine. 


\section{Regulating Molecular Testing}

According to the 1976 Medical Device Amendments to the Federal Food, Drug, and Cosmetic Act (FD\&C Act), in vitro diagnostics (IVDs), a broad category of products that includes molecular tests, are regulated by the FDA as devices. ${ }^{9}$ A laboratorydeveloped test (LDT) is a type of IVD that is designed, manufactured, and used within a single laboratory. LDTs differ from traditional laboratory tests, which are typically marketed commercially, distributed to several laboratories, and subject to FDA regulation per the FD\&C Act. Use of IVDs in a laboratory setting is regulated by CMS under the 1988 Clinical Laboratory Improvement Amendments. Historically, the FDA pursued a policy of enforcement discretion toward LDTs and, due to the simple nature and limited availability of the laboratory tests, has not enforced premarket review and other applicable FDA requirements. Due to advances in technology, such as next-generation sequencing (NGS), LDTs continue to evolve and multiply.

The FDA looks to play a larger role in regulating LDTs, although this has been met with criticism. In 2014, the FDA made a major push to regulate LDTs with the release of a planned regulatory framework and an ensuing public comment period. ${ }^{10}$ In July 2016, the FDA released 2 guidance documents on NGS-based tests. Stakeholders continue to voice concerns that the FDA review of LDTs would significantly slow innovation, reduce competition in the marketplace, and affect patient access to life-saving tests. ${ }^{11}$ As of January 2017, the FDA decided to delay finalizing a draft guidance on regulating LDTs; instead they released a discussion paper regarding the oversight of LDTs. ${ }^{12,13}$ The FDA finalized its guidance document for in vitro companion diagnostics in $\mathrm{Au}$ gust 2014 and released a second guidance document in July 2016. The FDA started to discuss nonrequired complementary diagnostics that provide significant information about the use of the drug, such as determining benefits and risks.

\section{Diagnostics and Financial Regulatory Issues}

Coverage and reimbursement for molecular diagnostics are ever-changing and often affected by emerging payment models. The complexity of coding diagnostics has soared, and technology assessments further complicate reimbursement. In payment models, test reimbursement is sometimes provided in a bundled amount that includes the test and other services. Particular to molecular tests is the Medicare 14-day rule, which requires laboratories to bill the hospital for the clinical laboratory services provided to the Medicare patient when services are ordered within 14 days of a patient's CMS covered discharge. Some argue that the regulation creates delays when ordering tests and impairs the ability to offer timely care, whereas others believe the delay in testing is designed to enhance revenue by the performing laboratory.

The Protecting Access to Medicare Act (PAMA) was signed in 2014, with a final rule implementing Section 216(a) released in June 2016. The law reworks the payment system for molecular tests. Under the rule, laboratories performing clinical diagnostic laboratory tests must report private payor payment rates and volume data. Medicare will then use these private insurer rates to calculate Medicare payment rates for laboratory tests under the Clinical Laboratory Fee Schedule beginning January 1, 2018. ${ }^{14}$

When there is a solid link in molecular testing and therapeutics, providers receive reimbursement for those specific tests. However, the complexity of reimbursement arises when those tests are embedded in a more comprehensive genomic profile. ${ }^{4}$ Furthermore, coverage for molecular diagnostic tests varies by payer. In early January 2016, Independence Blue Cross announced an agreement with NantHealth (Culver City, CA) to become the first major insurer to offer coverage for next-generation whole-genome sequencing. ${ }^{15}$ Some payers wait for diagnostics to be included in guidelines, such as those of NCCN or ASCO, to determine coverage. According to PAMA, CMS is authorized to consolidate the number of Medicare Administrative Contractors (MACs) that have authority to establish local coverage determinations and process claims for clinical diagnostic laboratory tests. Currently, there are 12 MACs that have this authority, the most notable of which is Palmetto GBA (Columbia, SC) and their Molecular Diagnostic Services (MolDX) Program. In the PAMA final rule issued in 2016, CMS did not exercise its discretion to consolidate the aforementioned authority among 1 to 4 MACs. Some argue that the coverage criteria pioneered by the MolDx Program set the "gold standard" for how the value of molecular diagnostics should be evaluated by all payers. ${ }^{16}$ Recognizing the data availability restrictions to determine clinical utility, the 
Winckworth-Prejsnar et al

MolDX Program uses a mechanism called Coverage with Data Development, which "may provide coverage for promising, but unproven diagnostic tests contingent on the submission of plans to conduct a clinical study that will generate additional data to support their safety, diagnostic performance, and most importantly, clinical utility." 17

Often, the clinical utility of a molecular test drives the coverage decision, yet a common evidentiary standard for demonstrating clinical utility is lacking. If common evidentiary standards are accepted, more consistency with reimbursement could be established. ${ }^{4}$ Frueh and Quinn ${ }^{18}$ proposed a 6-part framework, based on 6 key questions, that intends to frame and assess the importance of new diagnostic tests:

- Who should be tested and under what circumstances?

- What does the test tell us, that we did not know without it?

- Can we act on the information provided by the test?

- Does the outcome change in a way we find value in, relative to the outcomes obtained without the test?

- Will we act on the information provided by the test?

- If the test is to be used, can we afford it?

Under proposed Medicare guidelines for evaluating diagnostic tests, a diagnostic must pass 2 questions: (1) is the evidence adequate to determine that the use of the test provides more accurate diagnostic information, and if the test improves accuracy, and (2) is the evidence adequate to conclude that the improved accuracy will lead to better health outcomes? ${ }^{19}$ The answers determine whether the diagnostic has clinical utility from the Medicare perspective. Similarly, the Blue Cross Blue Shield Association criteria state that the scientific evidence must permit conclusions concerning the effect of the technology on health outcomes. ${ }^{1}$ The issue with these criteria is determining or defining scientific evidence that permits conclusions. Adequate evidence is a subjective concept and it is not intuitively obvious what level of evidence achieves public health or long-term health system value. ${ }^{1}$ Entrenched in the quest for a definition of adequate evidence are competing social objectives. The level of scientific evidence needed may have implications on how quickly new diagnostics are introduced for clinical use, while at the same time ensuring safety for patients by not exposing people to treatments or testing of uncertain benefit.

The Center for Medical Technology Policy's Green Park Collaborative, highlighted during the Policy Summit as a best practice, is a multistakeholder forum intended to develop evidentiary standards for clinical studies to inform coverage and reimbursement decisions. It has active involvement from public and private payers and includes patients, providers, regulators, industry, and other stakeholders. The Green Park Collaborative develops "effectiveness guidance documents" and provides specific recommendations on how to design studies intended to meet reimbursement or coverage standards to inform decisions by payers. ${ }^{1}$ These documents are analogous and complementary to FDA guidance and focused on design elements particularly relevant to reimbursement and clinical decision-making. ${ }^{20} \mathrm{Al}$ though many payers are involved in efforts such as the Green Park Collaborative, the collaborative lacks regulatory authority, so payers can choose whether to apply or adapt these documents accordingly. ${ }^{4}$

\section{Future Landscape of Molecular Testing}

Moving forward, panelists highlighted the need to understand molecular testing data in different and more novel ways. This might include efficient collection of data outside traditional conventional clinical trials, focusing on patient outcomes to effectively demonstrate clinical utility. Additionally, the uptake of molecular testing will rely on the proliferation of communication tools given to providers and patients to further explain biomarkers and targeted therapies. Lastly, a true collaboration of all stakeholders, including payers, is needed to make the considerable investment in resources to enhance the science of tumor biology within reimbursement paradigms that account for total cost of care.

\section{Biosimilars in Oncology: Developments, Trends, and Future Implications}

Over the past 2 decades, biologics revolutionized treatment options and supportive care management for patients with cancer. Biologic products, or biologics, are medical products made from various natural sources, including human, animal, or microorganism, and include vaccines, blood and blood components, and recombinant therapeutic proteins that 
prevent, mitigate, treat, or cure a range of diseases or medical conditions. ${ }^{21}$ In contrast to small molecular weight drugs, biologics are typically large, complex molecules that require strenuous processes for manufacturing and evaluating for safety, and therefore are often a high-cost component of cancer care. With an increase in approval of new biologics, coupled with the expanding use of existing biologic products, global spending on biologics continues to increase at a rapid pace. Biologics will account for nearly $20 \%$ of all global pharmaceutical sales in 2017, constituting roughly $\$ 221$ billion, and make up $45 \%$ of the latestage oncology global pipeline. ${ }^{22,23}$

The high and increasing cost of biologics is an area of concern for many stakeholders, because it may limit patient access to important and lifesaving therapies. Approval of highly similar biologics (biosimilars) is a key component of discussions regarding curbing rising healthcare costs and related access issues, partly because of their cost-savings potential within the oncology space. It is projected that biosimilar agents could cost $20 \%$ to $40 \%$ less than their reference product, the originator biologic, saving the US healthcare system anywhere from $\$ 44$ to $\$ 250$ billion between 2014 and 2024. ${ }^{24-26}$ Globally, it is estimated that 50 distinct biosimilars are currently in development, likely to increase competition in the global marketplace. Therefore, the evolution of biosimilars in the United States is of great interest to many different stakeholders, because these agents have the potential to lower costs and increase access without compromising health outcomes for patients with cancer.

In March 2015, 9 years after the first biosimilar was approved in Europe, the FDA approved the first biosimilar product in the United States, a milestone greatly anticipated within the oncology community. At the time of writing, a total of 4 biosimilars have been approved by the FDA, with filgrastim-sndz and infliximab-dyyb currently available in the US market. Although no approved anticancer therapeutic biosimilars are currently available in the United States, filgrastim-sndz is a supportive care biosimilar classed as a granulocyte colony-stimulating factor (G-CSF). It is approved for all indications of filgrastim to treat patients with (1) nonmyeloid malignancy receiving myelosuppressive chemotherapy with an intermediate- or high-risk of febrile neutropenia; (2) acute myeloid leukemia receiving induction or consolidation chemotherapy; (3) cancer undergoing bone marrow transplantation or peripheral blood progenitor cell collection and therapy; (4) severe chronic neutropenia; and (5) HIV infection. ${ }^{24}$

Although approval of the first biosimilars and numerous published FDA guidance documents further clarified requirements for biosimilar development and approval, complexities of interchangeability, substitution practices, extrapolation of scientific evidence, and naming procedures of biosimilars coming to market have moved to the forefront of the biosimilars debate. Additionally, pricing, cost impact, reimbursement, patient access, and a focus on pharmacovigilance will continue to influence the scope and scale of biosimilar uptake in the United States.

\section{Regulatory Overview of Biologics and Biosimilars}

A biologic product is approved by the FDA based on product-specific preclinical and clinical data under the Public Health Service Act (PHSA) and is used to treat various health conditions that might include cancer, multiple sclerosis, rheumatoid arthritis, and other, often chronic, diseases. The Biologics Price Competition and Innovation Act of 2009 (BPCI Act), part of the Patient Protection and Affordable Care Act (ACA), paved the way for the proliferation of biosimilars in the Unites States by granting the FDA the authority to create an abbreviated pathway for biosimilar approval. More specifically, the BPCI Act established an approval pathway under section $351(\mathrm{k})$ of the PHSA for biological products that are shown, among other things, to be highly similar to or interchangeable with an already approved FDAlicensed biological reference product with no clinically meaningful difference in terms of safety, purity, and potency from the reference product. ${ }^{27}$ The FDA outlines a stepwise approach for demonstrating biosimilarity, considering the totality of the evidence based on structural and functional characterization, animal data, human pharmacokinetic and pharmacodynamic data, clinical immunogenicity data, and any comparative clinical study data. ${ }^{28}$ The first biosimilar product available in the United States, filgrastim-sndz, was approved by the FDA based on review of evidence that included structural and functional characterization, animal study data, human pharmacokinetic and pharmacodynamic data, clinical immunogenicity data, and other clinical safety and effectiveness data demonstrating biosimilarity, 
Winckworth-Prejsnar et al

including a phase III noninferiority clinical trial known as PIONEER. ${ }^{29}$

Although biosimilars and their reference biologic products share many attributes, biosimilars have minor formulation or structural differences and will never be identical to their reference product, an important distinction from generic compounds of traditional small molecule medicine. Although biosimilars are approved through an abbreviated pathway, products must still meet high manufacturing standards and present sufficient evidence to demonstrate the product is safe and effective. ${ }^{30}$ In contrast to generic products, the designation of biosimilarity depends on the totality of evidence and permits reliance on existing scientific knowledge regarding the safety and effectiveness of the reference biologic product.

\section{Extrapolation}

According to the FDA, the potential exists in the $315(\mathrm{k})$ pathway for a biosimilar to be licensed for one or more conditions of use for which the reference product is licensed, referred to as extrapolation. Extrapolation is considered by the FDA on a case-by-case basis and requires sufficient justification to support a determination of biosimilarity for each condition of use for which licensure is sought. Extrapolation across indications for a biosimilar depends on several factors, including a common mode-of-action and receptor/ target/interaction, totality of the evidence showing comparability, acceptable safety profile without increased risks of immunogenicity, and clinical experience with the originator product to support the use of a biosimilar across indications. ${ }^{30,31}$ Extrapolation is not automatic, and scientific justification is required for each additional indication. Pharmaceutical biosimilar manufacturers generally design their biosimilar molecules with the expectation that they will adequately demonstrate the appropriate data for extrapolation. ${ }^{30}$ The rationale for extrapolation is the avoidance of unnecessary studies in the target population for efficiency, ethical reasons, and resource allocation. ${ }^{32}$ This approach is supported by extensive evidence in Europe, where many biosimilars were approved with extrapolation to all reference product indications. Traditionally, small molecule medicine relies heavily on phase III trials and randomized data to assess the safety and efficacy of a product. In contrast, approval of a biosimilar for one indication is used for extrapolation to other indications without the performance of clinical trials. Thus, from a provider perspective, the idea of extrapolation of biosimilars is a paradigm shift in how the biologic is developed, as well as in the clinical development and clinical use implications. ${ }^{30,31}$

The only off-label data considered in the NCCN Clinical Practice Guidelines in Oncology (NCCN Guidelines) is based on at least phase II studies that suggest benefit of the agent in a given disease setting. ${ }^{30,31}$ Dr. Crawford, chair of the NCCN Myeloid Growth Factors Panel, discussed NCCN category of evidence designation without clinical trial data for all indications of filgrastim-sndz. Within the NCCN Guidelines, although filgrastim-sndz is interchangeable for all indications with filgrastim, the panel concluded that the evidence for mobilization of allogeneic hematopoietic cell donors was not as strong as for other indications, and it was a given a category 2B designation. The early rollout of biosimilars in Europe, however, facilitated increased evidence (phase II studies and years of pharmacovigilance) beyond the FDA submission application to help inform guideline determinations for other indications..$^{30,31}$

Although selected biologics and biosimilars are used in the treatment of cancer, others serve as supportive care agents to reduce the adverse effects of cancer treatments such as chemotherapy and radiation. Panelists highlighted gaps in provider and patient perception of biosimilars used as supportive agents versus therapeutic agents. Often supportive care biosimilars have more adequate pharmacodynamic data markers (eg, hemoglobin, neutrophil count), helping to alleviate concerns for increased clinical validation. For therapeutic biosimilar agents, providers need to see the same degree of safety and lack of immunogenicity already proven by the adoption of supportive care biosimilar agents. Going forward, clinical experts caution about the need to focus on additional research for biosimilars moving from oncologic to nononcologic indications, particularly with respect to immunogenicity and safety. ${ }^{30}$ Ultimately, however, for biosimilars to be successful and to change the cost equation for patients, the principle of extrapolation needs to be applied broadly across disease states and stages. ${ }^{30}$

\section{Interchangeability and Substitution}

With the potential exposure of individual patients to the same drug multiple times over a lifetime, the topics of interchangeability and substitution 
continue to dominate the biosimilar discussion. An interchangeable biological product, in addition to meeting the biosimilarity standard, is expected to produce the same clinical result as the reference product in any given patient. The FDA can determine a biosimilar interchangeable with additional evidence demonstrating the biosimilar produces the same result in each patient and lacks a safety risk or diminished efficacy if the patient is switched between the biosimilar and reference biologic product. ${ }^{27}$ The FDA emphasizes that there is often a misconception that interchangeability requires a more rigorous standard of evidence. Rather, from the FDA perspective, there are different requirements and data submission under the BPCI Act to support a designation of interchangeability, not necessarily a higher standard..$^{30}$ In January 2017, the FDA released draft guidance on criteria for interchangeability, recommending one or more switching studies to show that patients can safely alternate between the biosimilar and reference product without diminished efficacy. ${ }^{33}$ To date, no biosimilars in the United States are approved as interchangeable.

According to the FDA, interchangeable products may be substituted for the reference product without the intervention of the prescribing healthcare provider. $^{34}$ In other words, substitution can occur where a prescribed reference biologic is replaced by an interchangeable biosimilar. Although the FDA only recently published draft guidance on interchangeability, 36 states have already debated legislation establishing standards for substitution of a biosimilar product to replace the reference biologic. ${ }^{35}$ For example, in 2015 alone, 11 states passed legislation limiting the substitution of biologics to biosimilars deemed interchangeable by the FDA, bringing the total number of states with laws restricting substitution of biologics to $18 .{ }^{36}$ Details regarding substitution legislation differ from state to state, but frequently have 3 common features:

- Substitution is only permitted if the biosimilar has been designated as interchangeable;

- Substitution may be prohibited if the prescribing physician has indicated a preference for the reference product; and

- The prescriber must be notified of any substitution made by the pharmacy. ${ }^{31,32}$

Panelists clarified that interchangeability and substitution practices only apply to biosimilars going through a pharmacy for distribution, often a small subset of all biosimilar products. Amgen Inc (Thousand Oaks, CA) notes that most of their biosimilar pipeline products are monoclonal antibodies, meaning that they generally must be administered by a doctor and will not go through a pharmacy for distribution. ${ }^{30}$ Additionally, it only applies to switching between the reference product and the biosimilar, not between 2 different biosimilars. The 2017 FDA draft guidance on interchangeability echoes discussions during the summit on the importance of switching studies to prove greater pharmacovigilance for interchangeability practices. ${ }^{30}$ Lastly, it is important to communicate to providers and patients that, although it is sometimes perceived, according to the FDA, a determination of biosimilar interchangeability is not a mark of greater approval than biosimilarity alone. ${ }^{30}$

\section{Naming}

Since the passage of the BPCI Act, the process of naming biosimilars has often been an area of disagreement among stakeholders. Policies around biosimilar naming is vital to pharmacovigilance, specifically the postmarketing monitoring of biosimilars and their reference products in terms of safety and efficacy. ${ }^{30}$ Much debate concerns whether the reference product and all corresponding biosimilars should use the same nonproprietary name, use the same nonproprietary name with a unique suffix, use the same nonproprietary name with a unique prefix, or use a different and unique nonproprietary name. Moreover, disagreement between stakeholders relates to whether the suffix or prefix should be random or convey meaning.

In August 2015, the FDA released a draft guidance for biosimilar naming, proposing a naming pathway that would use a unique 4-letter suffix following the product's nonproprietary name to differentiate between biosimilars and their reference products. ${ }^{37}$ The FDA solicited feedback on whether the 4-letter suffix should be randomly assigned or meaningful. Since the release of the $2015 \mathrm{draft}$ guidance, the FDA has taken a varying approach with respect to suffix meaning. In January 2017, the FDA finalized the Nonproprietary Naming of Biological Products: Guidance for Industry, which remained largely unchanged from the August 2015 draft guidance, proposing a meaningless, 4-letter suffix following 
Winckworth-Prejsnar et al

the product's nonproprietary name. ${ }^{38}$ Although the finalized guidance allows applicants to propose 10 suffixes, they cannot resemble the applicant's name or other FDA-designated nonproprietary name suffixes. The guidance also noted that the random suffixes will also be used for newly licensed and previously licensed reference biologic products. Although the final guidance outlined the intended use of the same nonproprietary name and suffix for interchangeable biosimilars, it did not specify the suffix format that will be used.

Panelists discussed how biosimilar naming policies that lack the same nonproprietary name or use a prefix/suffix that does not convey any meaning could potentially lessen the ability to monitor and track safety records, limiting the future pharmacovigilance of biosimilars. Moreover, such policies could also jeopardize the consistency with which biosimilars are correctly entered into electronic health record systems, thus further endangering the ability to track biosimilars and to ensure patient safety. From a patient perspective, having a memorable suffix would help alleviate concerns that the right medication is being prescribed by a provider or a pharmacy..$^{30}$ Overall, panelists agreed that consistent policies around naming of biosimilars are important for overall patient safety and cancer care moving forward.

\section{Cost, Alternative Payment Models Environment, and Reimbursement}

The 10 highest-expenditure drugs covered under Medicare Part B are biologics, representing nearly $50 \%$ of all Part B spending, highlighting why potential cost savings anticipated from a growing biosimilars market is of great interest to the oncology community. ${ }^{39}$ Current estimates predict biosimilars will be priced $20 \%$ to $40 \%$ less than reference biologic products. ${ }^{24}$ Competition in European countries, which introduced a legal pathway for biosimilars in 2004, show a reduction in healthcare costs, and many within the healthcare system are optimistic that increased commercialization of biosimilars will have a positive impact on access to lifesaving therapies for patients in the United States. Moving forward, opportunities for cost savings depend on the proliferation of several biosimilars approved for the same indication. ${ }^{30}$
Since NCCN last brought together key stakeholders for the 2013 Policy Summit on biosimilars, there has been a greater focus on value-based payment models to address increasing costs and access to high-quality healthcare. ${ }^{40}$ Coupled with a shift from fee-for-service payment to new value-based payment models, drug value-frameworks, formulary exclusions, and indication-specific pricing have emerged as strategies to define and improve value in cancer care. Panelists highlighted the significance in understanding these alternative payment trends and further exploring the implications they might have on the biosimilar landscape.

The 2016 CMS Medicare Physician Fee Schedule included a final payment rule grouping all biosimilars sharing the same reference product under a single Healthcare Common Procedure Coding System (HCPCS) code. Under this policy, all biosimilars sharing a HCPCS code will be reimbursed based in the volume-weighted average sales price. Stakeholders adamantly raised concerns that this outlined methodology for biosimilar reimbursement would discourage manufacturers from investing in additional indications, thus reducing the number of biosimilars available in the market. ${ }^{41}$ Moreover, some argued that this methodology will affect patient safety, reduce pharmacovigilance, influence other payer reimbursement policies, and threaten biosimilar sustainability. Panelists identified a number of remaining uncertainties regarding the future dynamics of the biosimilar marketplace. For example, the potential of biosimilars to be reimbursed at lower rates could discourage manufacturers from entering the biosimilar marketplace, consequently limiting the availability of biosimilars in the long run. Panelists cautioned that biosimilar prices will remain high without determination of interchangeability and several biosimilars approved for the same indication on the market, while recognizing that some integrated segments of the market might become more price-sensitive than others. ${ }^{30}$

\section{Future Landscape of Biosimilars}

Panelists highlighted a number of therapeutic oncology biosimilars currently in development within the trastuzumab (Herceptin) space, and ongoing phase III trials with bevacizumab (Avastin) and rituximab (Rituxan), in addition to ongoing supportive care biosimilars in development. ${ }^{30}$ As more therapeutic 
oncologic biosimilars come to market, increased evidence might help clarify issues regarding extrapolation, interchangeability, and substitution within the oncology community.

Panelists concluded the session by emphasizing the vital role that biosimilars play in the future of sustainable cancer care. Panelists predicted that patients, providers, payers, and legislators will increasingly have greater comfort and acceptance regarding the use of biosimilars. For uptake to occur, however, panelists emphasized that it will take a number of stakeholders involved to communicate the safety and importance of biosimilars within patient and provider communities. Lastly, panelists predicted that the science will improve in areas of molecular aspects and immunogenicity predictions, helping to drive competition, innovation, and development of future biologics and biosimilars.

\section{Conclusions}

With both biosimilars and molecular testing come challenges in ensuring patient access to innovation in cancer care while maintaining safe, effective, ethical, and affordable treatment options. Biosimilars and molecular testing face challenges in building adequate evidence regarding clinical utility, as well as safe and appropriate incorporation into clinical practice for patients with cancer. In order for molecular testing and biosimilars to achieve widespread adoption and coverage, stakeholders must rely on paradigm shifts away from a reliance on traditional clinical trials, further inclusion in guidelines and compendia in response to increased evidence, and improved education for providers and patients to ensure testing and use of biosimilars is implemented in a safe, efficient, and cost-effective manner.

\section{References}

1. Tunis $\mathrm{S}$. Clinical implications of biosimilars and molecular testing in oncology. Presented at the NCCN Policy Summit: Developments in Biosimilars and Molecular Testing; September 16, 2016; Washington, DC

2. Febbo P. Developments in molecular testing: benefits, risks, and challenges of application in cancer care. Presented at the NCCN Policy Summit: Developments in Biosimilars and Molecular Testing; September 16, 2016; Washington, DC.

3. Biomarker Tests for Molecularly Targeted Therapies: Key to Unlocking Precision Medicine. Washington, DC: The National Academies Press; 2016.

4. Molecular testing in oncology: effective approaches to maximize patient outcomes. Panel discussion at the NCCN Policy Summit: Developments in Biosimilars and Molecular Testing; September 16, 2016; Washington, DC.

5. IMS Institute for Healthcare Informatics. Innovation in cancer care and implications for health systems. Global oncology trend report. Available at: http://obroncology.com/imshealth/content/IMSH_Oncology_Trend_ Report_020514F4_screen.pdf. Accessed January 5, $201 \overline{7}$.

6. American Association for Cancer Research. Medicines in development for cancer. Available at: http://phrma-docs.phrma.org/sites/default/files/pdf/ oncology-report-2015.pdf. Accessed January 5, 2017.

7. Stanek EJ, Sanders CL, Taber KA, et al. Adoption of pharmacogenomic testing by US physicians: results of a nationwide survey. Clin Pharmacol Ther 2012;91:450-458

8. Hyman DM, Puzanov I, Subbiah V, et al. Vemurafenib in multiple nonmelanoma cancers with BRAF V600 mutations. N Engl J Med 2015;373:726-736

9. Engstrom PF, Bloom MG, Demetri GD, et al. NCCN molecular testing white paper: effectiveness, efficiency, and reimbursement. J Natl Compr Canc Netw 2011;9(Suppl 6):S1-16.

10. FDA and LDT's: should it "guidance" or "regulation"? Available at: http:// www.discoveriesinhealthpolicy.com/2014/10/fda-and-ldts-should-it-beguidance-or.html. Accessed January 7, 2017.

11. Quinn B. Inside Health Policy. Labs brace for change as FDA, CMS policies emerge. Available at: https://insidehealthpolicy.com/daily-news/ labs-brace-change-fda-cms-policies-emerge. Accessed January 7, 2017.

12. FDA holding off on finalizing regulatory guidance for lab-developed tests. GenomeWeb Web site. Available at: https://www.genomeweb.com/ molecular-diagnostics/fda-holding-finalizing-regulatory-guidance-labdeveloped-tests. Accessed December 20, 2016.
13. U.S. Food and Drug Administration. Discussion paper on laboratory developed tests (LDTs). Available at: http://www.fda.gov/downloads/ MedicalDevices/ProductsandMedicalProcedures/InVitroDiagnostics/ LaboratoryDevelopedTests/UCM536965.pdf. Accessed December 19, 2016.

14. Centers for Medicare \& Medicaid Services. Medicare will use private payor prices to set payment rates for clinical diagnostic laboratory tests starting in 2018. Available at: https://www.cms.gov/ Newsroom/MediaReleaseDatabase/Press-releases/2016-Press-releasesitems/2016-06-17.html. Accessed December 12, 2016.

15. Independence Blue Cross Newsroom. Independence Blue Cross becomes first major insurer to cover next-generation whole genome sequencing for a variety of cancers. Available at: http://news.ibx.com/independenceblue-cross-becomes-first-major-insurer-to-cover-next-generation-wholegenome-sequencing-for-a-variety-of-cancers/. Accessed December 8, 2016.

16. Hughes K. MolDX may be the norm, but is it the future? Available at: http://avalere.com/expertise/life-sciences/insights/moldx-may-be-thenorm-but-is-it-the-future. Accessed January 17, 2017.

17. Palmetto GBA. Molecular diagnostic program (MolDX®): coverage, coding, and pricing standards and requirements (M00106). Available at: http://palmgba.com/filestorage/moldx/MolDX_Manual.pdf. Accessed January 11, 2017.

18. Frueh FW, Quinn B. Molecular diagnostics clinical utility strategy: a sixpart framework. Expert Rev Mol Diagn 2014;14:777-786.

19. Centers for Medicare \& Medicaid Services. Proposed guidelines for evaluating diagnostic tests. Available at: https://www.cms.gov/medicarecoverage-database/details/medcac-meeting-details.aspx?NCDId=211\&n $c d v e r=1 \& N C A I d=85 \&$ NcaName $=$ Positron + Emission + Tomography +( FDG $\&$ id $=8 \% 2 F \& b c=$ gIAAAAAAAAAAAA $\% 3 D \% 3 D \& . \quad$ Accessed December 12, 2017.

20. Tunis SR. An overview of the green park collaborative-USA. Available at: http://www.cmtpnet.org/docs/resources/Tunis_Overview_of_GPCUSA.pdf. Accessed January 17, 2017.

21. U.S. Food \& Drug Administration. What are "biologics" questions and answers. Available at: http://www.fda.gov/AboutFDA/CentersOffices/ OfficeofMedicalProductsandTobacco/CBER/ucm133077.htm. Accessed December 10, 2016.

22. IMS Institute for Healthcare Informatics. The global use of medicines: outlook through 2017. Available at: http://www.imshealth.com/files/web/ IMSH\%20Institute/Reports/US_Use_of_Meds_2013/IIHI_Global_Use_ of_Meds_Report_2013.pdf. Accessed April 21, 2017. 
Winckworth-Prejsnar et al

23. IMS Institute for Healthcare Informatics. Global outlook for medicines through 2018. Available at: http://static.correofarmaceutico.com/ docs/2014/12/01/informe_ims.pdf. Accessed January 5, 2017.

24. Zelenetz AD, Becker PS. The role of biosimilars. J Natl Compr Canc Netw 2016;14:626-629.

25. Miller S. The $\$ 250$ billion potential of biosimilars. Available at: http://lab. express-scripts.com/insights/industry-updates/the-\$250-billion-potentialof-biosimilars. Accessed December 18, 2016

26. Mulcahy AW, Predmore Z, Mattke S. The cost savings potential of biosimilar drugs in the United States. Available at: http://www.rand.org/ pubs/perspectives/PE127.html. Accessed January 10, 2017.

27. U.S. Congress, H.R. 3590-686, Biologics Price Competition and Innovation Act of 2009. Available at: http:/www.fda.gov/downloads/ Drugs/ GuidanceComplianceRegulatoryInformation/ ucm216146.pdf. Accessed December 8, 2016.

28. U.S. Food \& Drug Administration. CDERLearn: FDA overview of biosimilar products. Available at: http:/www.fda.gov/Training/ ForHealthProfessionals/default.htm. Accessed December 22, 2016.

29. US Food \& Drug Administration. FDA approves first biosimilar product Zarxio. Available at: www.fda.gov/Drugs/InformationOnDrugs/ ApprovedDrugs/ucm436648.htm. Accessed April 14, 2017.

30. Biosimilars: readiness for application in clinical cancer care. Panel discussion at the NCCN Policy Summit: Developments in Biosimilars and Molecular Testing; September 16, 2016; Washington, DC.

31. Crawford J. Biosimilars in oncology: developments, trends, and future implications. NCCN Policy Summit: Developments in Biosimilars and Molecular Testing; September 16, 2016; Washington, DC.

32. European Medicines Agency. Concept paper on extrapolation of efficacy and safety in medicine development. Available at: http://www.ema. europa.eu/docs/en_GB/document_library/Scientific guideline/2013/04/ WC500142358.pdf. Accessed April 21, 2107.

33. Considerations in demonstrating interchangeability with a reference product: guidance for industry. Available at: http://www.fda.gov/ downloads/Drugs/GuidanceComplianceRegulatoryInformation/ Guidances/UCM537135.pdf. Accessed January 19, 2017.
34. Biosimilars: questions and answers regarding implementation of the Biologics Price Competition and Innovation Act of 2009: guidance for industry. Available at: https://www.fda.gov/downloads/drugs/guidances/ ucm444661.pdf. Accessed April 21, 2017.

35. National Conference of State Legislators. State laws and legislation related to biologic medications and substitution of biosimilars. Available at: http://www.ncsl.org/research/health/state-laws-and-legislation-related to-biologic-medications-and-substitution-of-biosimilars.aspx. Accessed January 5, 2017.

36. IMS Institute for Healthcare Informatics. Medicines use and spending in the U.S.: a review of 2015 and outlook to 2020. Available at: https:// morningconsult.com/wp-content/uploads/2016/04/IMS-Institute-US Drug-Spending-2015.pdf. Accessed January 5, 2017.

37. U.S. Food \& Drug Administration Nonproprietary naming of biological products; draft guidance for industry; availability. Available at: https:// www.federalregister.gov/articles/2015/08/28/2015-21383/nonproprietarynaming-of-biological-products-draft-guidance-for-industry-availability. Accessed November 18, 2015.

38. U.S. Food \& Drug Administration. Nonproprietary naming of biological products: guidance for industry. Available at: http://www.fda. gov/downloads/Drugs/GuidanceComplianceRegulatoryInformation/ Guidances/UCM459987.pdf. Accessed January 13, 2017

39. U.S. Government Accountability Office. Medicare Part B: expenditures for new drugs concentrated among a few drugs, and most were costly for beneficiaries. Available at: http://www.gao.gov/products/GAO-16-12 Accessed December 22, 2017

40. National Comprehensive Cancer Network. Evolving Policy Issues in Oncology - Revisiting Biosimilars and Molecular Testing. Available at: https://www.nccn.org/professionals/meetings/oncology_policy_program/ pdf/2013 evolving_policy_issues_in_oncology_summit_summary.pdf. Accessed February 1, 2017.

41. Quinn B. Regulation of biosimilars and molecular testing in oncology. Presented at the NCCN Policy Summit: Developments in Biosimilars and Molecular Testing; September 16, 2016; Washington, DC.

\section{See JNCCN.org for supplemental online content.}

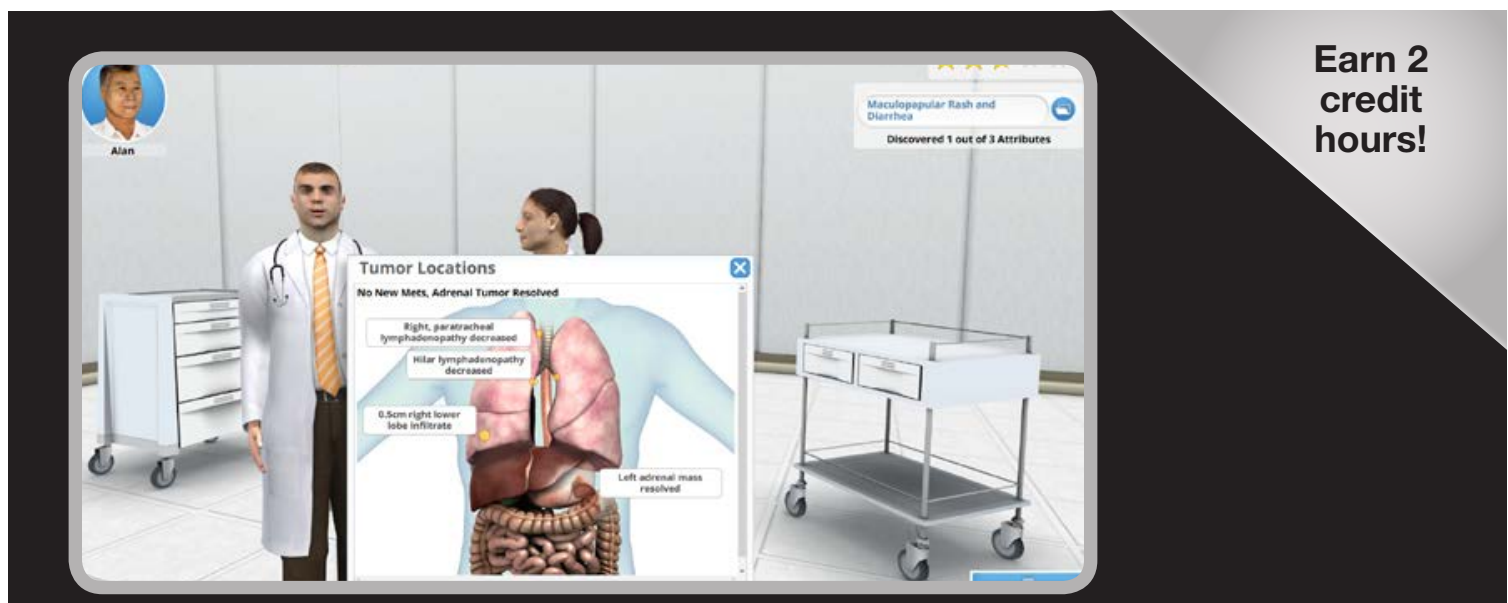

ADVANCED NON-SMALL CELL LUNG CANCER PATIENT MANAGEMENT SIMULATOR

Brought to you by the National Comprehensive Cancer Network ${ }^{\circledR}$ (NCCN ${ }^{\circledR}$ )

Powered by Syandus Simulation Technology

education.nccn.org/nsclc-simulator

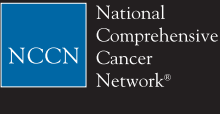

